\title{
Una terminología de teatro para organizar el patrimonio cultural universitario*1
}

\author{
Catalina Naumis Peña ${ }^{1}$ [orcid.org/0000-0003-3152-3958], Ariel Alejandro Rodríguez García ${ }^{2}$ \\ [orcid.org/0000-0001-5779-0371], Hugo Alberto Guadarrama Sánchez ${ }^{3}$ [orcid.org/0000-0002-1917-8015] \\ 1. Universidad Nacional Autónoma de México, Ciudad de México. \\ naumis@unam.mx \\ 2. Universidad Nacional Autónoma de México, Ciudad de México. \\ ariel@iibi.unam.mx \\ 3. Universidad Nacional Autónoma de México, Ciudad de México. \\ bibliotekallica@comunidad.unam.mx
}

\begin{abstract}
Resumen: El presente estudio tiene como objetivo identificar los términos funcionales en la comunicación sobre el tema del teatro para un sistema de información de espacios culturales universitarios en idioma español. En cuanto a la designación terminológica existe un conjunto de zonas, áreas, medios, instrumentos y herramientas para intercambiar entre tramoyistas, técnicos de sonido e iluminación, músicos, actores y artistas que deben ser manejadas por los responsables de la operación de los espacios y de las autoridades que programan actividades. La metodología se fundamentó en la búsqueda de los términos referentes al espacio teatral y la confrontación con el Art \& Architecture Thesaurus (Getty Research Institute) Esta categorización permitió delimitar el universo del teatro de acuerdo a sus conjuntos y sus respectivos elementos, para establecer una estructura que jerarquiza el espacio teatral, con la finalidad de sustentar la indización y recuperación del sistema de información en el que se insertará. Finalmente, se consultaron a los especialistas y autoridades para validar la propuesta.
\end{abstract}

Palabras claves: sistemas de información, terminología del teatro, patrimonio cultural universitario, recuperación de información, difusión cultural universitaria

\begin{abstract}
The objective of this study is to identify the functional terms in the communication on the theme of theater for an information system of university cultural spaces in the Spanish language. Regarding the terminological designation, there is a set of zones, areas, means, instruments and tools to exchange between stagehand, sound and lighting technicians, musicians, actors and artists that should be managed by those responsible for the operation of the spaces and the authorities that schedule activities. The methodology was based on the search of the terms referring to the theatrical space and the confrontation with the Art \& Architecture Thesaurus (Getty Research Institute) This categorization allowed to delimit the theater universe according to its sets and their respective elements, to establish a structure that hierarchizes the theatrical space, in order to sustain the indexing and recovery of the information system in which it will be inserted. Finally, the specialists and authorities were consulted to validate the proposal.
\end{abstract}

Keywords: information systems, theater terminology, university cultural heritage, information retrieval, university cultural dissemination

\section{Introducción}

Las Industrias Culturales y Creativas (ICC) significan la manifestación intrínseca de la capacidad de la humanidad para pensar, crear, innovar y diseñar productos, experiencias y servicios, generando un valor económico dentro de un contexto comercial (Peris-Ortiz, Cabrera-Flores y Serrano-Santoyo, 2019, p. 3). Sin embargo, en el ámbito universitario, el patrimonio cultural complementa el modelo de formación de los estudiantes, comunidad universitaria y público en general y no se considera ese valor económico como prioritario, ni se le otorga la categoría de industria.

La Universidad Nacional Autónoma de México (UNAM) tiene un inmenso patrimonio cultural (entre otros valores, el campus central de la Ciudad Universitaria es patrimonio de la humanidad, reconocida por la UNESCO en el año 2007) que pone al servicio de su tarea educativa, de investigación y de difusión de la cultura. El hecho de disponer de varios campus en muchas ciudades de México y del extranjero plantea entre otros retos, manejar la información concerniente a los espacios destinados a la cultura.

Por tanto, la Coordinación de Difusión Cultural de la UNAM ha desarrollado un sistema de información sobre los espacios y activos culturales: Sistema de Información para el Registro

\footnotetext{
${ }^{1}$ Esta comunicación forma parte del proyecto apoyado con recursos PAPIIT IT400318
} 
Universitario de Espacios y Activos Culturales (SI-RUEyAC), con la asesoría del Instituto de Investigaciones Bibliotecológicas y de la Información (IIBI). El rol de la información y la comunicación sobre el particular tiene como objetivo prioritario expandir el uso y acceso cultural de la comunidad universitaria hacia el desarrollo del individuo para concretar su proyecto de aprendizaje, a través de la programación de actividades. Entre los problemas a resolver para el correcto funcionamiento del sistema de información está el lenguaje utilizado para indizar y recuperar la información.

De acuerdo con el SI-RUEyAC a la cabeza del árbol de dominio que abarca su sistema están los principales términos que agrupan los espacios universitarios existentes, que es bastante más amplio que sólo el teatro especializado: teatro multiuso, recintos especializados, explanadas y foros al aire libre. Las razones para encabezar los temas a través de esta propuesta son las necesidades de este sistema de información, ya que, en los planteles de educación media, de educación superior e institutos de investigación abundan los teatros multiusos o auditorios. Entre los recintos especializados existen teatros en los que se presentan obras para público en general y deben cumplir con los requisitos que se exigen en ellos. Una de las ideas que subyacen con el sistema es que sirva para evaluar la capacidad de los teatros multiusos o auditorios para ser usados en representaciones profesionales.

En el caso del presente trabajo se analizarán únicamente las relaciones jerárquicas de los términos del teatro bajo los recintos especializados teatrales y no en el sentido amplio de teatro. Esta será una parte del tesauro que se elabora para sustentar el SI-RUEyAC. Para cumplir con esta parte del trabajo, se busca identificar los términos cuyos nodos conceptuales son funcionales en la comunicación sobre el tema del espacio teatral, sin incluir en este caso, los mecanismos tecnológicos asociados. El conocimiento y discusión del uso terminológico busca asegurar un significado dominado por el grupo destinado a programar el uso de los espacios culturales universitarios. La clave de la comunicación en un sistema de información es que el emisor y el receptor comparten no solamente las mismas palabras, sino que también les asignan el mismo significado.

Las obras teatrales, los ciclos, las ferias del arte y los festivales, en principio son ideados y conceptualizados como una vía para la apertura de contenidos hacia un público con necesidades culturales específicas, dichos contenidos suelen ser planificados y difundidos a través de los medios impresos y digitales. No obstante, los contenidos requieren de una plataforma, escenario o estructura para desplegarse y desarrollarse, es decir de un espacio actoral (Pavis, Patrice, 1998, p. 169).

Los espacios en donde se presentan las actividades y expresiones culturales poseen diversos componentes mecánicos, eléctricos y electrónicos orientados a la creación de atmósferas y dinámicas en las puestas en escena, dado que cada proyecto u obra teatral tiene características particulares por su naturaleza temática y público. En un sentido técnico, en cuanto al uso de la palabra, la diversidad terminológica permite nombrar el conjunto de zonas, áreas, medios, instrumentos y herramientas para intercambiar información con los responsables de los espacios, por parte de los tramoyistas, técnicos en sonido e iluminación, músicos, actores y artistas; aunque en ocasiones existe la posibilidad de conflictos a partir de la confusión y omisión de elementos, como resultado de esa misma diversidad terminológica ya sea de oficio, disciplinaria o profesional en el ámbito teatral (Dinulović, 2015, p. 4).

En el teatro se utiliza un lenguaje específico en cada acción, fenómeno o actividad realizada para montar un espectáculo en un espacio donde transcurre la interacción entre actores y público, con la participación de diferentes profesionales especializados en el espectáculo programado. Cada especialista usa las denominaciones comunes a su área, que a veces no coinciden con las otorgadas por otros especialistas a la misma situación. En este complejo plano de interacción concurren además las autoridades universitarias y los responsables de los espacios en general, que deben tomar las decisiones referentes a los usos espaciales y los activos correspondientes para cumplir con la actividad teatral. 
El lenguaje coadyuva a las diversas aristas disciplinarias y administrativas que se relacionan en dicho contexto, para obtener un mejor entendimiento y comprensión acerca de cada elemento que se implementa en el teatro, como pueden ser las divisiones entre las áreas del público y las interiores, en donde interactúan músicos, cantantes, actores, tramoyistas o maquinistas, carpinteros, escenógrafos, utileros, vestuaristas y demás, cada uno de ellos comunicándose entre sí, a veces con confusiones e imprecisiones para el conjunto de los profesionales y técnicos que conviven día a día (Theatre projects consultants, 2016, p. 2).

El estudio se centra en la terminología del espacio teatral y en las estructuras en donde se lleva a cabo la actividad histriónica y áreas destinadas al público, no se incluyen términos relativos a las estructuras internas de las obras dramáticas, como por ejemplo las entradas y salidas de los actores frente al público o tras bambalinas, en forma física o a través únicamente de la voz, diferencias entre tipos de personajes o los movimientos de los actores en el escenario (Meierkhold, 1994, p. 152). Los términos que se incluyen están relacionados con los objetivos del sistema de información desarrollado y es a través de la especificación del dominio del espacio teatral donde transcurren que serán de utilidad en la indización y recuperación de la información.

En principio y para los fines de este estudio se plantea esa representación del dominio del teatro como espacio y se agregan los componentes que lo integran como complemento de ese espacio y la función de apoyar un evento teatral. La terminología de un dominio se conforma con el vocabulario integrado de, por lo menos, dos anillos: uno que podría denominarse como nuclear, y otro afín (Barité, 2017, p. 470).

\section{Metodología}

La investigación terminológica se realiza en un ámbito de conocimiento, buscando la representación de los fenómenos que ocurren al interior. Por ello cada término es investigado en su vehiculación de significado. El análisis de un término implica el conocimiento de su uso oral y también escrito para obtener la garantía de uso por la comunidad de hablantes.

La metodología se fundamentó en la búsqueda de los términos referentes al espacio teatral, verificados en primera instancia en imágenes y videos demostrativos sobre estos. Una vez entendido el espacio se investigaron las designaciones en las obras especializadas como los glosarios, los diccionarios, las enciclopedias, los vocabularios controlados existentes e inclusive hasta los trabajos académicos universitarios en idioma español, confrontados con los usos en la práctica.

El éxito del sistema de información dependerá de una estructura terminológica entendible para los usuarios de éste. En el caso del teatro el vocabulario controlado más difundido y usado es el Art \& Architecture Thesaurus (Getty Research Institute). Este tesauro (Tesauro del P. G.) será continuamente consensado en relación a los términos a incorporar a la base de datos que se construye. Se consulta tanto la versión en inglés como la traducción al español. El primer acercamiento a los términos de teatro se realizó a partir de esta herramienta lingüística, como se habrá de explicar a través de ejemplos donde se detecta incompatibilidad con el sistema que se construye. Las equivalencias en el español del Getty con las usadas por la comunidad de hablantes del ámbito de difusión cultural universitario para referirse a los aspectos y características del teatro en México no son comprensibles en todos los casos, basta mencionar que la designación que en México se conoce como "sala del teatro" en el Getty se la designa como "espacio de auditorio".

La terminología que se estudia estará integrada en un tesauro que comprende los espacios culturales universitarios en general. En los planteles de enseñanza se generaliza como espacio cultural al auditorio. Si el término utilizado en los recintos profesionales de teatro es "espacio de auditorio" se confunde la comunicación. De esta manera, no quedará especificada la conformación de la sala de teatro al interior del teatro y se presentaría en la misma categoría del espacio de auditorio que es mucho más general. Por ejemplo, un espacio de auditorio puede comprender el lugar al aire libre donde colocar sillas para comodidad de los asistentes. 
La preferencia del lenguaje en idioma español está determinada por el uso del sistema donde quedará insertada la terminología. La metodología se centra en la selección y extracción de términos que representan el teatro y sus componentes en información localizada en bibliografía en español, algunas veces confrontada con el inglés, agregando información obtenida de internet que comprende tanto videos como páginas web. Los términos obtenidos son contrastados en las entrevistas realizadas a los responsables de los teatros que han participado en el levantamiento de información llevado a cabo en los espacios, para recabar la información que contendrá el sistema de información en desarrollo sobre espacios y activos culturales universitarios.

Por supuesto, la finalización del proceso en cada etapa de categorización de la terminología que se propone es sometida a la evaluación de los especialistas de difusión cultural quienes, como se explicó en líneas arriba, propusieron el desarrollo del sistema de información sobre los espacios y activos culturales de la UNAM.

Por ello es a partir de dichas entrevistas e interacciones con las autoridades de difusión cultural, los técnicos de teatro y especialistas vinculados al ámbito universitario, que se hace un nuevo análisis y extracción de términos sobre el tema, para explorar los términos validados por la comunidad y las nuevas relaciones a establecer como funcionales en la comunicación, que asegure la comprensión por los diferentes tipos de usuarios a los que se destina el sistema de información en construcción.

\section{Relación de términos entre el Art \& Architecture Thesaurus (Getty Research Institute) y las necesidades del SI-RUEyAC}

El propósito del Tesauro de Arte y Arquitectura del Paul Getty (Tesauro del P. G.) es constituir un recurso estructurado con el fin de mejorar el acceso a la información referente al arte y a los recintos culturales. Sin embargo, la organización de sus metadatos y enlaces tiende a combinar distintos conjuntos temáticos al momento de realizar la recuperación de la información, provocando cierta confusión en su consulta cuando se carece de conocimientos sobre los temas en ámbitos muy específicos. El propósito del SI-RUEyAC es elaborar una herramienta, con la finalidad de programar las actividades culturales en una universidad que posee un número muy alto de espacios donde realizarlas. Si bien la base de datos incluye la infraestructura en cuanto a equipo, esta parte de la terminología, no se incluye en el presente estudio.

En el análisis de los usos terminológicos utilizados en el SI-RUEyAC y luego de revisar la traducción de los términos al español del Tesauro del P. G., así como los términos en inglés, a pesar de que sus términos cumplen con los estándares de valor para la catalogación; no podrían ser utilizados en el contexto explicado más arriba, dado que sus transliteraciones al español no corresponden del todo a los significados de los espacios y activos culturales contemplados. Del mismo modo la representación esquemática de la terminología teatral carece de algunos elementos y excede de otros, como por ejemplo los vinculados al teatro griego, de manera que, hay mayor presencia en el Tesauro del P. G. de los términos que representan a los teatros al aire libre.

La UNAM carece de teatros al aire libre (se ha detectado uno) y si bien hay teatros especializados, en general son teatros multiusos o auditorios que se deben adaptar a diferentes funcionalidades. Por esta razón las características de los teatros especializados deben quedar bien definidas a través del vocabulario controlado que se construye, para hacer las comparaciones necesarias a la hora de la programación.

En un primer acercamiento al Tesauro del P. G. se observa que las traducciones al español siguen el razonamiento utilizado en inglés, esto se percibe por el uso de la palabra teatro que en inglés es más amplia que en el español y sin embargo se usa en la traducción, según la tradición en inglés.

La primera jerarquía donde se asienta el teatro como espacio o edificio es precedido por la clasificación obtenida de la copia del Tesauro del P. G. y que parte de Built Environment, con las 
siguientes meronimias, miniature theaters, puppet theaters y por último theater elements:

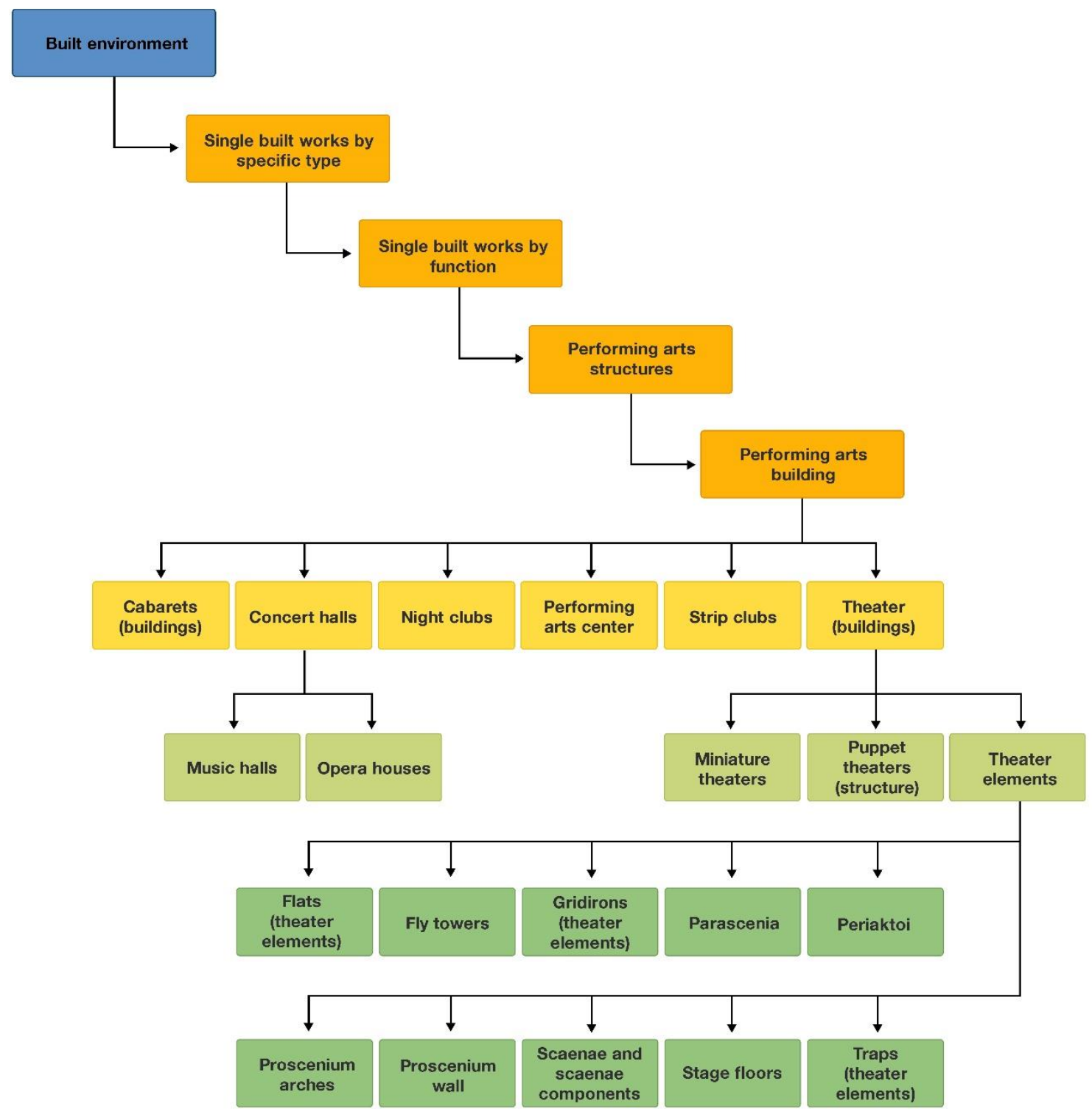

Figura 1: Ubicación jerárquica del Teatro como espacio en el Tesauro del P.G. Fuente: Adaptación de Luis Enrique Sánchez Rodríguez, a partir de la extracción del tesauro del P. G. con fecha del 18 de mayo del 2019.

Como se puede observar la relación jerárquica para llegar a Teatro como espacio es demasiado extendida. En la traducción al español se presenta el mismo esquema terminológico. En el Tesauro del P. G. <performing space building>, es decir el espacio escénico del teatro se comparte con los cabarets o las discotecas y los clubs de streaptease. Tampoco interesan las meronimias establecidas para los teatros como espacios, porque los elementos del teatro comparten relaciones con los teatros en miniatura y los teatros de marionetas. En el SiRUEyAC los elementos del teatro como espacio (delimitado como tal) deben ser separados en áreas: pública, técnica, escénica y administrativa. Las necesidades del SiRUEyAC apuntan a una estructura diferente para usuarios no catalogadores y que necesitan claridad y sencillez a la hora de registrar o consultar la información. Se parte de una taxonomía de la UNAM, donde cada sitio ingresado está relacionado con la dependencia donde se encuentra el espacio escénico, porque el objetivo prioritario es el aprovechamiento de los espacios disponibles en la universidad

De esta manera, el Tesauro del P.G. como herramienta de indización y recuperación podría no ser idónea para fines de un ámbito de conocimiento más especializado en el mundo de habla hispana, 
puesto que en su mayor parte se encuentra constituido por términos en inglés refiriéndose a un vocabulario de muchísima amplitud, mucha más de la necesaria para estructurar el sistema de información que se construye.

Más allá de la operatividad para el SI-RUEyAC, si el objetivo principal del Tesauro del P. G. es el de proporcionar una terminología sobre los lugares culturales para las diversas necesidades, muy a pesar de su objetividad en cuanto a la representación de las entidades; requiere sin duda de una revisión a fondo para la equivalencia a un español común al ámbito hispanoamericano. De no ser así, el uso del tesauro como herramienta ocasionaría una problemática latente al momento de ser implementado por los catalogadores y especialistas en los museos, las bibliotecas de arte y los archivos en hispano-américa.

De acuerdo con la institución que lo sustenta su tesauro crece constantemente mediante las aportaciones de los proyectos de investigación, por lo que resulta conveniente el hecho de colaborar con el fin de conformar una perspectiva iberoamericana. Por esa razón el universo de los espacios y activos culturales de la UNAM debe delimitarse conceptualmente a partir de la creación de conjuntos o categorías que permitan establecer subcategorías con los términos en el español usado en México, basados en la garantía oral y literaria de los expertos hablantes del español, con el fin de establecer las equivalencias en términos significativos de nuestro idioma para ampliar y mejorar el recurso en línea del Getty Research Institute.

\section{Análisis terminológico del teatro como espacio cultural}

El teatro es la representación de ideas (texto), en un determinado espacio (escena), a través de la acción del cuerpo (expresión corporal), la cara (gesto) y la integración de la palabra (fonética), con la utilización de los códigos socialmente aceptados (o no), para estimular los sentidos y obtener reacciones (comunicación), hacia el medio o entorno (Psicosocial), estas reacciones van desde la reflexión hasta la pura y simple diversión, entretenimiento, recreación y esparcimiento (Saavedra, 2018).

Al transcurrir en un espacio en el que concurre la representación teatral con el público que lo observa, es necesario contar con las características adecuadas para el impacto del trabajo actoral frente al público. Ello significa que la programación de actividades debe considerar que los espacios propuestos deben de contar con los elementos que permitan la interacción y el lenguaje de unos y otros para el transcurso ordenado del proceso de presentación del espectáculo.

De manera que, se parte de un listado de términos obtenidos de programas de trabajo, textos sobre la difusión teatral, bitácoras de presentación de obras y textos producidos en México sobre la actividad teatral. Estos elementos de análisis son parte de la garantía literaria que opera en los términos usados sobre el particular. De acuerdo a Barité (2011), "la garantía literaria proporciona los términos de referencia y el control del vocabulario les otorga la configuración definitiva y las relaciones" (p. 141).

Cada uno de los términos es confrontado en diccionarios de la especialidad y cuando las definiciones son amplias, se incluyen las acepciones que interesan al proyecto. Cada término es acompañado de una definición y cuando éstas no son claras para el sistema de información se incluyen notas de alcance, que explican el significado otorgado (Bails, 1802), (Plazola, 1996, p. 137), (Pavis, 1998, p. 169), (Romero y Pardo, 2003), (Zacarías, 2003, p. 263 y 264), (Pérez y Gardey, 2008), (Ferrera, 2009, p. 856, 1341 y 1456), (Especialidad de escenografía: Escuela Superior de Arte Dramático de Sevilla, 2011), (Consejo Nacional de la Cultura y las Artes, 2013), (Laiz, 2013), (Zertuche y Borrego, 2015), (Gaitán, Melina, 2016), (Glosarios servidor, 2016), (Theater projects, 2016), (Quizlet, 2019), (Departamento de composición ETSAB-UPC. Cuarto de control de iluminación, 2019) y (Real Academia Española, 2019).

La estructura que precede al teatro es el organismo del cual depende en la estructura de la UNAM. En general, los teatros profesionales que son el tema tratado en este trabajo se ubican directamente bajo la Coordinación de Difusión Cultural. Una vez que se recopilaron la generalidad de los términos y sus definiciones fueron agrupados bajo teatro, en las categorías mencionadas anteriormente: área pública externa, área pública interna, área escénica, área administrativa y área 
técnica Ello con el fin de poder delimitar el universo del teatro de acuerdo a sus conjuntos y sus respectivos elementos, para establecer una estructura que jerarquice el espacio teatral, relaciones de equivalencia y asociativas entre descriptores que expresan cierta afinidad semántica entre conceptos distintos, con la finalidad de sustentar la indización y recuperación del sistema de información en el que se insertará. Finalmente, el punto de vista más influyente y fundamental será el de los especialistas en el tema para validar y obtener un producto intelectual de calidad, capaz de responder a las necesidades de otras comunidades interesadas en el tema del teatro en el idioma español.

En base al análisis realizado sobre el tema del teatro se hace la siguiente propuesta para jerarquizar los términos que organicen la indización del SI-RUEyAC:

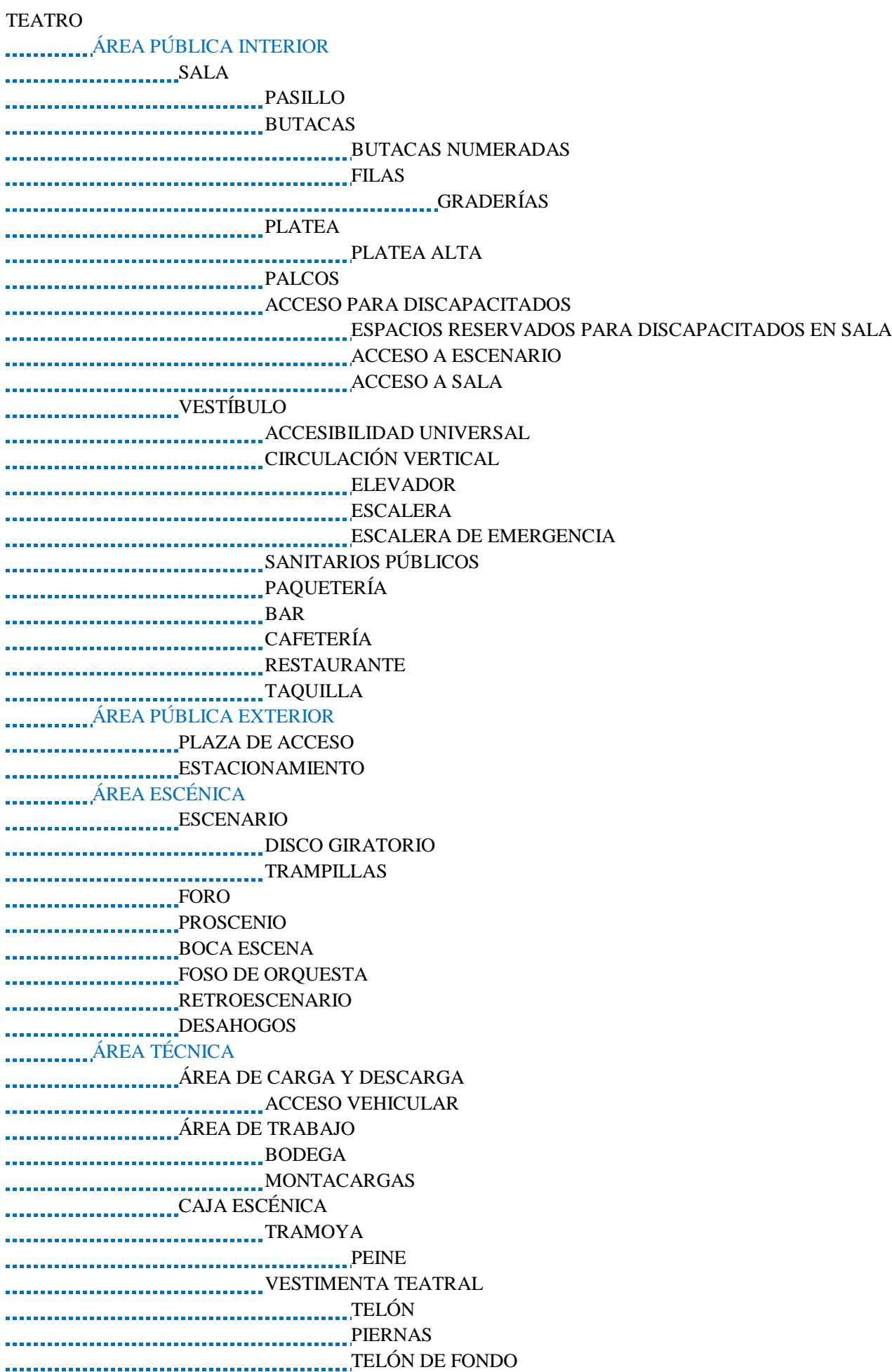




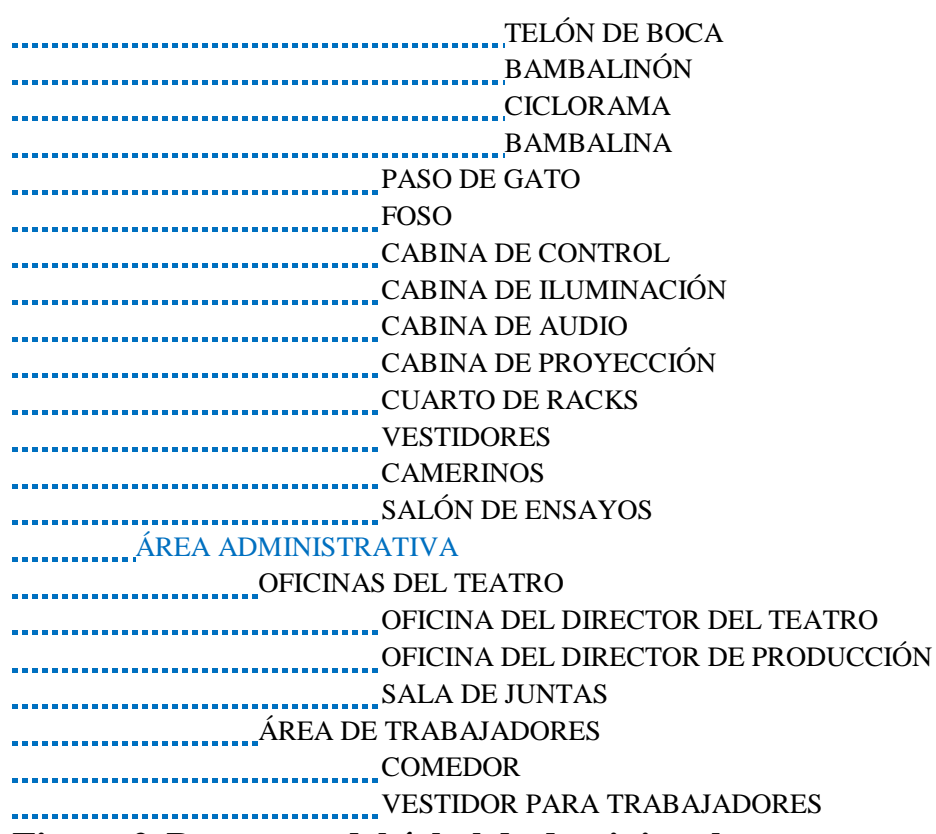

Figura 2. Propuesta del árbol de dominio sobre teatro para la base de datos del SI-RUEyAC.

\section{Discusión de los resultados}

La agrupación en áreas del teatro ayudará posteriormente a la organización de los otros grupos de recintos que deberán utilizarse para complementar los espacios culturales que posee la universidad. Sin duda, los auditorios no contarán con un desarrollo tan amplio de las áreas en que se distribuyen los términos del teatro, pero esa generalidad permitirá considerar aspectos menores que también se presentan en los auditorios. Por ejemplo, la programación exige conocer la estructura del área administrativa que gestiona los espacios en cada dependencia o las posibilidades del área técnica para acceder desde el exterior directamente al escenario, porque en el escenario no se contempla la comunicación exterior, o tener conocimiento de la existencia de un vestíbulo o plaza de acceso donde se pueda programar un brindis.

El área pública interna del dominio (figura 2) está dividida en Sala y Vestíbulo. Sin embargo, el acceso para discapacitados está incluido en la sala, aunque contempla las facilidades que puedan existir para acceder al escenario o al vestíbulo que son elementos que pertenecen a otras categorías. Las decisiones se adoptan de acuerdo a un área, pero en el tesauro se relacionarán con las otras que intervienen en el uso de los espacios.

Otros detalles que se observan es que no todos los teatros disponen de vestidores, cuyo sentido en este sistema de información es el lugar donde se agrupan las vestimentas utilizadas en las diferentes presentaciones llevadas a cabo, a diferencia de los camerinos que son más privados o de los vestidores de los trabajadores que se incluyen bajo la categoría del área de trabajadores. Otro término que sobresale es la vestimenta teatral que no está relacionada con la vestimenta que usan los actores, sino que se relaciona con el escenario y los telones.

Si bien existe una categoría general que es el área externa, también en el área técnica se incluye un área externa, pero exclusivamente relacionada con el área técnica porque es el área de carga y descarga.

Cómo se observa la estructura básica jerarquizada de los términos del teatro es sencilla y directa para facilitar la organización de la información incorporada al sistema, a diferencia del Tesauro del P. G. cuya estructura involucra términos innecesarios en el SI-RUEyAC.

\section{Consideraciones finales}

La terminología final estará insertada en un sistema de información que incluye todo tipo de espacio cultural y los activos de infraestructura que incluyen, donde se puedan programar actividades universitarias, no únicamente de espectáculos como el teatro. 
En el medio universitario mexicano se incluyen teatros (referidos a los teatros profesionales en el que se puede presentar una obra teatral), salas de conciertos y auditorios, además de espacios al aire libre como foros y explanadas, que se puedan adaptar a representaciones, como es el caso del carro de comedias.

El sistema de información en la etapa del proyecto que se desarrolla se alimentará usando un tesauro que se genera para indizar y establecer las categorías a manejar, con la finalidad de organizar la taxonomía básica, usando el software MultiTes versión 2018.10.30u. Sin embargo, a futuro se piensa integrar la terminología del tesauro en una ontología que se construirá para incrementar el potencial del sistema y hacerlo más funcional, por lo que, desde ahora, se incluyen definiciones de cada término para los elementos que se requieran a futuro.

Las ontologías incluyen una gran riqueza de relaciones entre términos, y ello exige un cuidado especial del análisis para aprovechar el presente estudio y explicitar los conceptos incluidos en el dominio analizado, con la finalidad de formalizarlos y que las computadoras puedan comunicarse entre sí, con éxito y sin ambigüedad, a fin de acrecentar la posibilidad de realizar inferencias (Barber, 2018, p. 14).

\section{Referencias}

Avita, A. (2011). Teatro para principiantes. México: Porrúa.

Barber, E., Pisano, S., Romagnoli, S., de Pedro, G., Gregui, C., Blanco, N., \& Mostaccio, M. (2018). Metodologías para el diseño de ontologías Web. Información, cultura y sociedad, 39, 13-36. Recuperado https://www.google.com/url?sa=t\&rct=j\&q=\&esrc=s\&source=web\&cd=1\&ved=2ahUKEwiqioD L5bDiAhUDnawKHTM1DHMQFjAAegQIABAC\&url=http\%3A\%2F\%2Fppct.caicyt.gov.ar\%2 Findex.php\%2Fics\%2Farticle\%2Fdownload\%2F14453\%2F45454575759139\&usg=AOvVaw2R 23M9rGsXAY1L7c-S57X2

Barité, M. \& Rauch, M. (2017). EL árbol de dominio como herramienta en organización del conocimiento: aplicación al área de educación superior. En Perspetivas de investigação em representação $e$ organização do conhecimento: Atualidade $e$ tendencias. Recuperado de http://sci.uc.pt/eventos/atas/comunicacoes/isko2017/isko2017_468 477.pdf

Barité, M. (2011). La garantía literaria como herramienta de revisión de sistemas de organización del conocimiento: modelo y aplicación. (Tesis doctoral). Universidad de Granada. Recuperado de http://digibug.ugr.es/handle/10481/17583

Consejo nacional de la cultura y las artes (2013) El escenario: lugar de trabajo en equipo. [PDF]. Recuperado de http://www.adtres.cl/files/4814/2543/5438/el_escenario_vol3.pdf

Dinulović, R. (2015). Space in the 20th Century Theatre: 1. Theatre and Architecture. SEE J Archit Des. 2015, 1-6. Recuperado de http://www.id-press.eu/seejad/article/view/seejad.2015.10006/pdf

Elevador. (2019). En Real Academia Española. Recuperado de https://dle.rae.es/?w=diccionario

Escenario. (2019). En Glosarios de términos especializados de las Ciencias, las Artes, las Técnicas y la Sociedad Alicante. Recuperado de https://glosarios.servidor-alicante.com/teatro/escenario

Escuela Superior de Arte Dramático de Sevilla. (2011). Glosario de términos. Sevilla, España: Especialidad de escenografía. Recuperado de http://esadsevillaescenografia.blogspot.com/2011/01/glosariode-terminos.html

Ferrera, J. (2009). Glosario ilustrado de las artes escénicas. España: Ediciones y Gráficas Solapas.

Gaitán, M. (2016, mayo 23). Historia de la arquitectura teatral. [Video]. Recuperado de https://www.youtube.com/watch?v=qSL5YWf6iGk

Laiz, M. (2013). El teatro. [Sitio web]. Recuperado de https://es.slideshare.net/lululululululu/las-partes-delteatro

Luneta. (1802). En Diccionario de Arquitectura Civil. Recuperado de http://enciclopedia.us.es/index.php/Luneta_(DACBB)

Meierkhold, V. (1994). El actor sobre la escena: diccionario de practica teatral. México: UAM: Grupo editorial gaceta.

Pavis, P. (1998). Diccionario del teatro. Barcelona; México: Paidos.

Pérez, J. \& Gardey, A. (2008). Definición de Rack. Recuperado de https://definicion.de/rack/

Peris-Ortiz, M., Cabrera-Flores, R. \& Serrano-Santoyo, A. (2019). Cultural and creative industries: A path to Entrepreneurship and Innovation. Suiza: Springer. Recuperado de https://doi.org/10.1007/9783-319-99590-8

Plazola, A. (1996). Enciclopedia de arquitectura Plazola. $\quad$ Estado de México: Plazola Editores. 
Romero, M. \& Pardo, A. (2003). Diseño de interior de teatros. (Tesis de licenciatura). Universidad del Istmo. Recuperado de http://glifos.unis.edu.gt/digital/tesis/2003/8515.pdf

Saavedra, R. (2018). Terminología básica del teatro. [Sitio web]. Recuperado de http://eurekateatro.ve.tripod.com/grupoeurekateatro/id6.html

Sutherland, A. (2019). Partes del teatro. [Diapositivas]. Recuperado de https://quizlet.com/85707091/partes-del-teatro-flash-cards/

Theatre projects (2016). Partes de un teatro. [PDF]. Recuperado de http://theatreprojects.com/files/pdf/resources_partsofatheatrebuilding_ES.pdf

Universitat Politécnica de Catalunya Barcelonatech. (2010). Cuarto de control de iluminación. Barcelona, España: Departamento de composición ETSAB-UPC. Recuperado de http://espaciosescenicos.org/Cabina-de-control-de-iluminacion

Zacarías, P. (2003). La arquitectura de los teatros veracruzanos durante el Porfiriato. México: Universidad Veracruzana.

Zertuche, C. \& Borrego, A. (2015, septiembre 8). Partes del Teatro. [Video]. Recuperado de https://www.youtube.com/watch?v=UKoC1O-SjOQ 